\title{
Power amplification characteristics of ultrafast pulses after the NPR port of Yb-doped fiber laser in all normal dispersion configuration
}

\author{
Pradeep K. Gupta, C. P. Singh, A. J. Singh, S. K. Sharma, P. K. Mukhopadhyay, K. S. Bindra and S. M. Oak \\ Solid State Laser Division \\ Raja Ramanna Centre for Advanced \\ Technology Indore, India-452013 \\ pkm@rrcat.gov.in
}

\begin{abstract}
The power amplification characteristics of all-fiber Yb-doped double clad fiber amplifier seeded by the pulses extracted after the nonlinear polarization rejection (NPR) port of all-normal dispersion (ANDi) mode-locked Yb-doped fiber oscillator is reported. $21.8 \mathrm{~W}$ of amplified average power at 37 $\mathrm{MHz}$ repetition rate was obtained at pump power of $35 \mathrm{~W}$ corresponding to $\sim 62 \%$ pump to signal power conversion efficiency. The single pulse energy of the amplified mode locked pulses was $\sim 590 \mathrm{~nJ}$. The effect of pre-amplifier signal strength on temporal and spectral profiles of amplified pulses were investigated experimentally. It was found that though stronger signal strength at the input of the power amplifier is useful to increase the threshold for onset of amplified spontaneous emission (ASE), however, it may lead to severe distortion in temporal profile after compression due to enhanced nonlinearity. We conclude that there is an optimum signal strength for generation of clean and smooth ultrashort pulses with low ASE contribution with in the operating range of the power amplifier. The compressed pulse duration of the amplified pulses was measured to be $280 \mathrm{fs}$.
\end{abstract}

Keywords-All normal dispersion, mode locked, fiber amplifier, Yb-doped fiber laser.

\section{INTRODUCTION}

Ytterbium ( $\mathrm{Yb}$ ) doped mode-locked fiber laser in all normal dispersion (ANDi) configuration has attracted a great deal of current interest due to its simplicity in construction and configurability with all-fiber integration. Further, the shape of the mode-locked pulses from the ANDi laser is dissipative soliton type which can tolerate a large variation of gain and loss and hence highly suitable for energy scaling in external amplifier. Since its first demonstration in 2006 [1], considerable progress has been made during the recent past to understand the pulse shaping dynamics in ANDi laser as well as to improve its performance [2-4]. However, the pulses from most of these systems exhibit large amount of side-lobes when they are compressed to femtosecond duration and the pulse quality degrades further on amplification [5]. One of the reasons for poor pulse quality from the ANDi laser is that in most of the systems the output is taken either before or at the nonlinear polarization rejection (NPR) port in order to extract maximum pulse energy, though, the influence of nonlinearity like self phase modulation (SPM) on the spectra of the pulses is very strong at those locations. Recently, we have shown that by extracting the pulses after the nonlinear polarization rejection port in ANDi Yb-doped fiber laser can provide a clean seed source which is considerably resistant to distortion on amplification as compared to that from the NPR-port and can be compressed into femtosecond regime without any significant side-lobes in the pulse profile [6]. It would be interesting to study the high power amplification characteristics of such mode locked pulses.

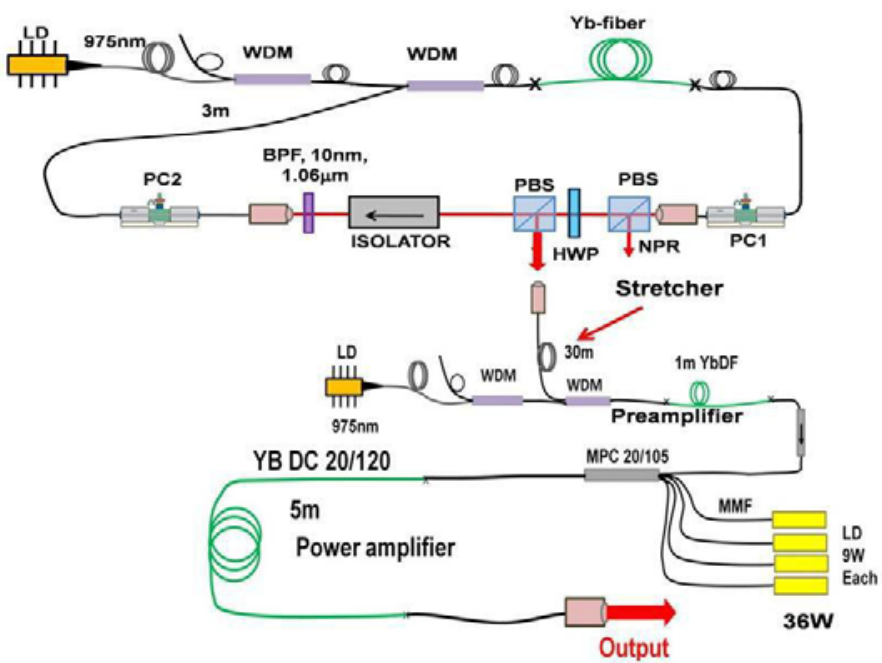

Figure 1: All-normal dispersion Yb-doped mode locked fiber oscillator-amplifier set-up.

In this paper, we report power amplification of pulses extracted after the NPR port of the mode locked Yb-doped ANDi laser. Mode locked pulses were amplified into Ybdoped double clad fiber (Yb DCF) amplifier. Around 21.8 W average amplified signal power with $35 \mathrm{~W}$ coupled pump power was obtained at fundamental repetition rate of $37 \mathrm{MHz}$. Spectral and temporal characterization of the power amplifier output was carried out for different input signal strength. In general, larger signal strength is desirable for power amplification to keep the ASE contribution low. However, strong signal strength leads to severely distorted pulse profile 
due to increased nonlinearity. We found an optimum signal strength for clean and smooth compressed pulses while keeping low ASE contribution.

\section{ALL NORMAL DISPERSION YTTERBIUM DOPED FIBER OSCILLATOR: GENERATION OF CLEAN PULSES}

The construction of the ANDi Yb-doped fiber laser is shown schematically in Fig.1. The oscillator is comprised of a $70 \mathrm{~cm}$ long single clad single mode Yb-doped fiber (YbDF) with mode field diameter of $6.0 \mu \mathrm{m}$. It was pumped in-core by a single mode fiber (SMF) coupled laser diode (LD) at $976 \mathrm{~nm}$ with the help of a 980/1060 WDM combiner.
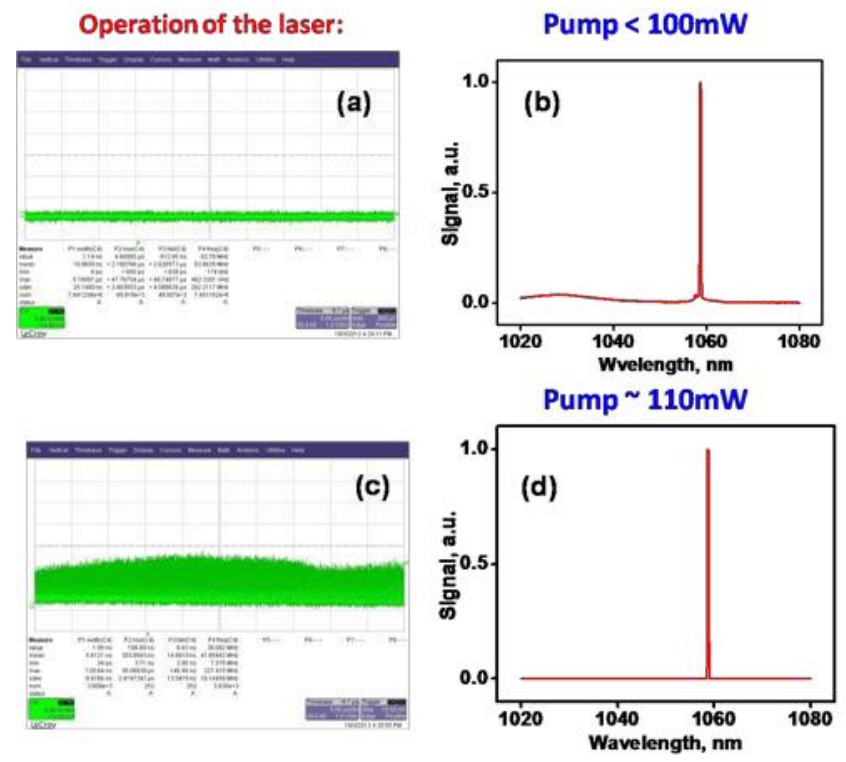

Pump $250 \mathrm{~mW}$
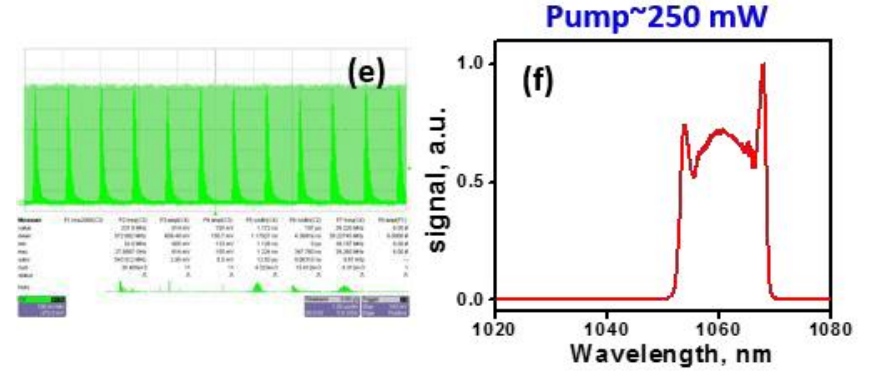

Figure2. Oscilloscope traces (a, c \& e) and laser spectrum (b, d \& f) in CW, QML \& cwML regime respectively.

The maximum pump power delivered in the core of the YbDF was $\sim 330 \mathrm{~mW}$. One end of the YbDF was spliced to the output port of the WDM and at the other end; a standard SMF (HI1060, $105 \mathrm{~cm}$ long) was connected. At the signal port of the WDM a $300 \mathrm{~cm}$ long SMF was spliced. The free ends of the two SMFs are connected to in-fiber collimators (COL1 and COL2). The total cavity length including the free space between the collimators was $\sim 570 \mathrm{~cm}$. A polarizing beam splitter (PBS1) is placed near COL1. The PBS1 in combination with the two infiber polarization controllers (PC1 and PC2) attached to the SMFs acts as the fast saturable absorber based on nonlinear polarization rotation. A fraction of the circulating power is coupled out from the cavity at PBS1 as the nonlinear polarization rejection. An output (OP) coupler is implemented after the PBS1 with the help of a half wave plate (HWP) and a polarizing beam splitter (PBS2). The polarization transmission axes of PBS1 and PBS2 are kept parallel to each other and by adjusting the orientation of the c-axis of the HWP with respect to the PBSs the output coupling ratio from the OP port can be varied for a wide range. For example when the c-axis of HWP is kept parallel to the polarization transmission direction of the beam splitters no output occurs through the OP port and the NPR port acts as the only output port for the laser, while orienting it at some other angle output also occurs through the OP port. In our experiment the c-axis of the HWP was oriented to $\sim 15^{0}$ with respect to the polarization transmission direction of the beam splitter which was found optimum for extracting the maximum pulse energy through the $\mathrm{OP}$ port. A bulk optical isolator (ISO) was placed in the free space for unidirectional ring cavity operation and a narrow band interference filter (BPF) with $10 \mathrm{~nm}$ bandwidth and peak transmission at 1060 $\mathrm{nm}$ is placed after the isolator for self starting and stable modelocking operation with a central wavelength around $1060 \mathrm{~nm}$. The net dispersion in the oscillator was estimated to be $\sim 0.12 \mathrm{ps}^{2}$. The laser can be easily mode-locked by adjusting the PC1 and PC2 and output can be taken either through the NPR port or OP port by orienting the c-axis of the HWP parallel or at $15^{0}$ to the

polarization transmission direction of the PBSs. We denote these two configurations of the laser as the 'NPR port setup' and 'OP port setup' respectively. In the OP port setup when HWP is oriented at $15^{\circ}$ to maximize the output from this port leakage signal from NPR port is also measured for comparison.

The evolution of mode locking in the oscillator segment is shown in Fig.2. The laser had a threshold $\sim 70 \mathrm{~mW}$ and operates in continuous wave (CW) mode upto $\sim 100 \mathrm{~mW}$ pump power. Fig. 2a and $2 \mathrm{~b}$ show the oscilloscope trace for $\mathrm{CW}$ operation and the corresponding lasing spectrum respectively. On increasing the pump power, the laser operates in Q-switched mode-locking (QML) regime. Fig. 2c and 2d show typical oscilloscope trace and spectrum of the QML lasing regime. However, when the pump power is increased beyond $240 \mathrm{~mW}$ stable self-starting continuous wave mode locking (cwML) is achieved and a stable train of modelocked pulses with a repetition rate of $37 \mathrm{MHz}$ is observed as shown in Fig. 2(e). As a signature of ultrashort pulses, the spectrum of the cwML pulses becomes broad as depicted in Fig. 2(f). The operational characteristics of the

ANDi oscillator is described in Fig. 3. Fig. 3(a) shows the variation of the output power from the 'NPR port setup' and the

corresponding mode-locking regimes as a function of the pump power. Since CW operation is observed over a very short pump power above threshold, it is not mentioned in Fig.3a. It can be seen from Fig.3 (a) that the laser operates in the QML regime until the pump power is below $240 \mathrm{~mW}$. However, when the pump power is increased beyond $240 \mathrm{~mW}$ stable self-starting continuous wave mode locking (cwML) is achieved and a stable train of mode-locked pulses with a repetition rate of $37 \mathrm{MHz}$ is observed. A maximum average power of $90 \mathrm{~mW}$ was obtained at pump power of $300 \mathrm{~mW}$ corresponding to pulse energy of $\sim 2.4 \mathrm{~nJ}$. The corresponding spectral profile of the mode-locked 
pulses was recorded with the help of an optical spectrum analyzer (Agilent 86142B) and is shown in Fig. 3(b). It can be seen that the spectra is the usual hare-head shaped [3] with large peaks at the edges. The root mean square (rms) spectral width

$\left(\Delta \lambda_{\text {rms }}\right)$ was measured to be $\sim 5.54 \mathrm{~nm}$. It is to be noted from

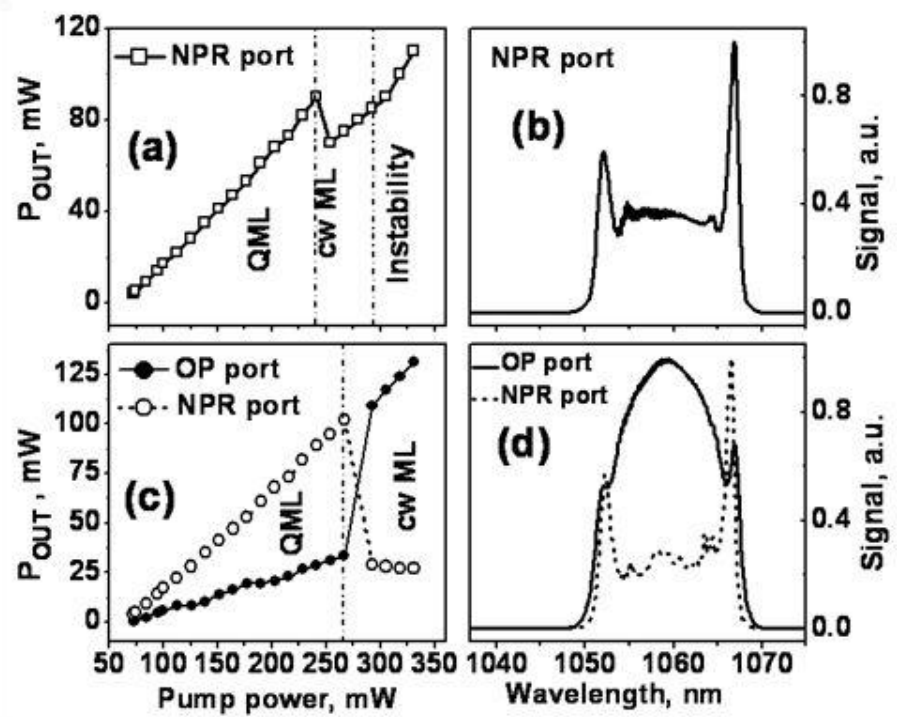

Figure.3.(a) and (b): operational and spectral characteristics of the oscillator in 'NPR port setup',(c) and (d): operational and spectral characteristics of the oscillator in ' $O P$ port setup'.

Fig.3(a) that as the pump power is increased beyond $300 \mathrm{~mW}$, instability in the mode-locked pulses takes place and stable cw

mode locking could not be obtained. The performance of the laser in 'OP port setup' is shown in Fig.3(c) and (d). The solid circles in Fig.3(c) shows the variation of the output power from the OP port as a function of the pump power. The power leaking-out through the NPR port in this setup is also shown in the same figure (open circles). It can be seen from Fig. 3(c) that the threshold for $\mathrm{cw}$ mode-locking is increased slightly ( $270 \mathrm{~mW})$ due to the coupling loss of the OP port and most of the output occurs through the NPR port in the QML regime. However in cwML regime there is a sudden increase in the power through the OP port and the output power through the NPR port is reduced drastically. The laser was in stable cwML regime even at the maximum operating pump power of $330 \mathrm{~mW}$ delivering $\sim 130 \mathrm{~mW}$ of average power. This corresponds to 3.5

$\mathrm{nJ}$ of pulse energy which is around $45 \%$ higher than that obtained from the 'NPR port setup'. The solid line in Fig. 3(d)

shows the spectral profile of the mode locked pulses through the OP port. It can be seen that the spectra is smooth and dome shaped with considerably diminished peaks at the edges. The rms spectral width was measured to be $\sim 4.8 \mathrm{~nm}$. The dashed line in the figure shows the spectra of the pulses leaking through the NPR port which is qualitatively similar in shape to Fig. 3(b) with a nearly same spectral width $\left(\Delta \lambda_{\text {rms }} \sim 5.55 \mathrm{~nm}\right)$.

Pulses from the oscillator are highly chirped with a measured duration of $5 \mathrm{ps}$. Around $10 \mathrm{~mW}$ of signal from the OP port was coupled to the stretcher fiber. The stretcher is made of $30 \mathrm{~m}$ long SMF which increases the pulses duration to around $30 \mathrm{ps}$. The pre-amplifier setup consists of a $1 \mathrm{~m}$ long
Yb doped SMF pumped in-core (400mW, 976nm) by a fiber coupled LD. A small scale amplification characteristics of the mode locked pulses obtained from the OP- and NPR-port have been studied into single clad single mode YbDF amplifier [6]. It was found that in contrast to NPR-port, the spectra of the pulses from the OP port is considerably resistant to distortions on amplification and can be compressed in the femtosecond regime (156 fs) without any significant side-lobes and hence can serve as an excellent seed source for further power amplification. Therefore, we have used seed signal only from OP-port for power amplification.

\section{POWER AMPLIFICATION OF ULTRASHORT} LASER PULSES

Power amplifier segment is designed based on prior simulations incorporating the interplay of seed signal strength, pump power, amplifier fiber length and the peak ASE power in ultrafast $\mathrm{Yb}$ DCF amplifier using a commercial simulation software [9]. An optimum choice of parameters for negligible ASE contribution is obtained. We have considered the amplification of signal power at $1060 \mathrm{~nm}$ co-propagating with the pump wavelength at $975 \mathrm{~nm}$ in the Yb-doped fiber. The growth of ASE, for each direction of propagation and their back-action on the local excited-state population due to wavelength-selective amplification and reabsorption can be modeled by the following rate equations involving the population inversion density $N_{2}(\mathrm{z})$, the pump field $P(\mathrm{z})$, the signal field $P_{\mathrm{s}}(\mathrm{z})$ and the ASE $P_{\mathrm{f}}(\mathrm{z})$ at a given longitudinal position $z$ along the fiber under the assumption of purely homogeneous broadening as [7]:

$$
\begin{aligned}
& \frac{d P(z)}{d z}=-\eta p \sigma_{a p} P(z)\left[N_{t o t}-(1+\delta) N_{2}(z)\right] \\
& \frac{d P_{S}\left(z, v_{S}\right)}{d z}=\eta_{S}^{\sigma} \sigma\left(v_{S}\right) P_{S}\left(z, v_{S}\right)\left[\left(1+\alpha\left(v_{S}\right)\right) N_{2}(z)-\alpha\left(v_{S}\right) N_{t o t}\right]
\end{aligned}
$$

$$
\frac{d P_{f}^{ \pm}(z, v)}{d z}= \pm \eta_{s} \sigma_{e}\left(v \underset{\mid+h v \delta v N}{\left.i P_{f}(z, v)\left[(1+\alpha(v)) N_{2}(z)-\alpha(v) N_{t o t}\right]\right]}\right.
$$

Here, $\boldsymbol{\eta}_{\mathrm{p}}$ and $\boldsymbol{\eta}_{\mathrm{s}}$ are the overlapping factors of the pump and signal respectively within the fiber core, $P(z)$ is the power of the pump, $P_{\mathrm{S}}(z, v)$ is the power spectral density of the injected co-propagating signal and $P_{\mathrm{f}}^{ \pm}(\boldsymbol{z}, v)$ is that for the copropagating and counter-propagating ASE. $N_{\text {tot }}$ is the dopant concentration, $N_{2}(z)$ is the metastable level density population. $\boldsymbol{\sigma}_{\mathrm{ap}}$ and $\boldsymbol{\sigma}_{\mathrm{ep}}\left(\mathbf{v}_{\mathrm{p}}\right)$ are the pump absorption and stimulated emission cross section. $\delta=\sigma_{\mathrm{ep}}\left(\boldsymbol{v}_{\mathrm{p}}\right) / \boldsymbol{\sigma}_{\mathrm{ap}}$ is the ratio between the stimulated emission and absorption cross section at the pump wavelength, and $\boldsymbol{\alpha}\left(\mathbf{v}_{\mathrm{s}}\right)=\boldsymbol{\sigma}_{\mathrm{as}}\left(\mathbf{v}_{\mathrm{s}}\right) / \boldsymbol{\sigma}_{\mathrm{es}}\left(\mathbf{v}_{\mathrm{s}}\right)$ is the ratio between the cross section of the signal absorption and stimulated emission. $\boldsymbol{\alpha}\left(\mathbf{v}_{\mathrm{s}}\right)=1$ for an ideal three-level system, and 0 for a pure fourlevel scheme. $\alpha(v)$ and $\sigma_{\mathrm{e}}(v)$ are the same for the ASE wavelength. In the ASE power expression, the factor $\boldsymbol{h} \boldsymbol{v} \boldsymbol{\delta} \boldsymbol{v}$ is the noise power corresponding to one photon per mode in 
bandwidth $\delta \boldsymbol{v}$; if the two polarizations can can be propagated in the fiber, the noise power becomes $2 \boldsymbol{h} \boldsymbol{v} \boldsymbol{\delta} \boldsymbol{v}$.

Eqs (1)-(3) are solved numerically using a commercial simulation software [8] for following parameters: core diameter $=20 \mu \mathrm{m}$, inner cladding diameter $=125 \mu \mathrm{m}, N_{\text {tot }}=$ $8.510 \mathrm{~m}$, ap $=2510 \mu \mathrm{m}$, ep $=21.710 \mu \mathrm{m}$, as $=$

$0.44810 \mu \mathrm{m}$, es $=2.6210 \mu \mathrm{m}$ and $=0.8 \mathrm{~ms}$. The simulation results are summarized in Fig.4. Fig. 4(a) shows the computed interdependence of signal strength, output power and pump power for a fixed gain fiber length whereas Fig. 4(b) shows the interdependence of signal strength, output power and length of gain fiber for a fixed pump power. Thus Fig.4 provides a guide line for selecting optimum length of the gain fiber depending on the available pump power and signal strength to obtain maximum amplified power with low ASE contribution. Figure 4 shows the simulation results for a wide range of input signal strengths for less than 1\% ASE contribution. It can be seen from Fig. 4(a) that the signal strength should be increased proportionally with the pump power in order to obtain maximum output power with minimum ASE signal for a fixed fiber length of $2 \mathrm{~m}$. Optimum length of the gain fiber required for a given signal strength at a fixed pump power of $40 \mathrm{~W}$ is shown in Fig. 4 (b). It can be seen that lower signal strength requires longer gain fiber to obtain the maximum output power with low ASE content.
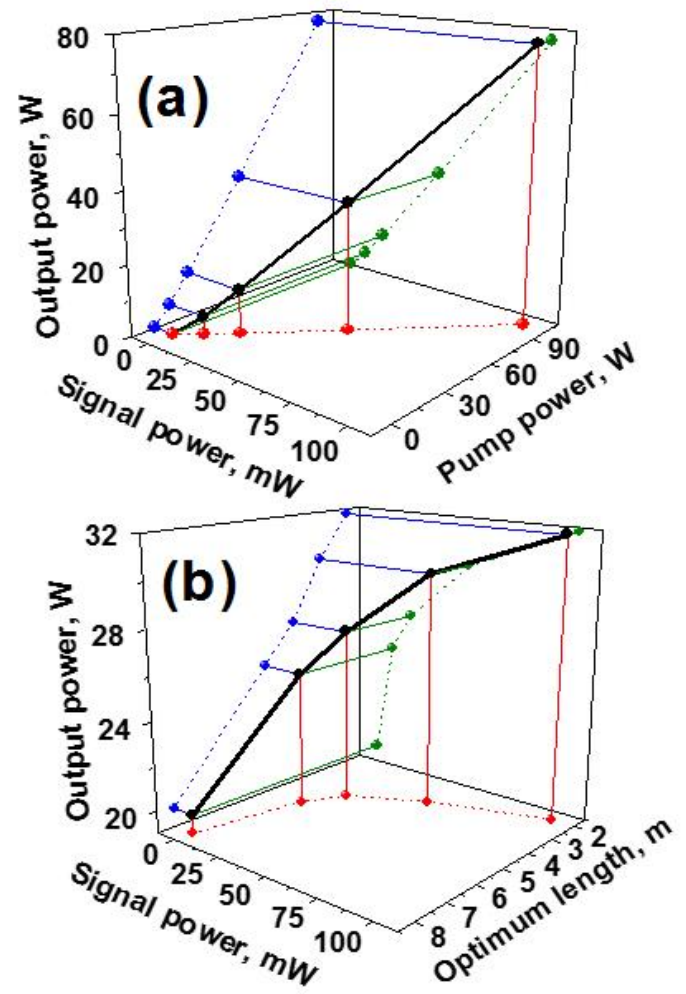

Figure 4: (a) Interdependence of the signal, pump and output power for low $(<1 \%)$ ASE contribution for a fixed fiber length of $2 \mathrm{~m}$ (b) Interdependence of optimum fiber length, signal strength and amplified power with less than 1\% ASE at a fixed pump power of $40 \mathrm{~W}$.
Power amplifier is designed in the lab based on the simulation results. The power amplifier segment is shown in Fig. (1). It is comprised of a $(6+1) \mathrm{x} 1$ multiple pump combiner (MPC) with signal feed through, a Yb-doped doubled clad fiber with $20 \mu \mathrm{m}$ core diameter and $125 \mu \mathrm{m}$ inner clad diameter (YDF-20/130-VIII, Nufern) and 4-nos of high power multimode fiber (core diameter $105 \mu \mathrm{m}$, N.A. 0.15) coupled laser diodes at $976 \mathrm{~nm}$ with $9 \mathrm{~W}$ of output power each. The MPC combines the pump beam from the high power laser diodes for cladding pumping of YbDCF and the signal from the preamplifier (via an isolator) to the core of the YbDCF for power amplification. Considering the insertion losses from isolator and MPC the signal strength coupled to the power amplifier can be varied in the range of $5 \mathrm{~mW}-60 \mathrm{~mW}$ by varying the preamplifier gain. The length of the YbDCF is kept fixed $\sim 5 \mathrm{~m}$ for complete absorption of the pump beam and the signal strength is adjusted to obtain low ASE contribution and clean temporal profile of the amplified pulses.
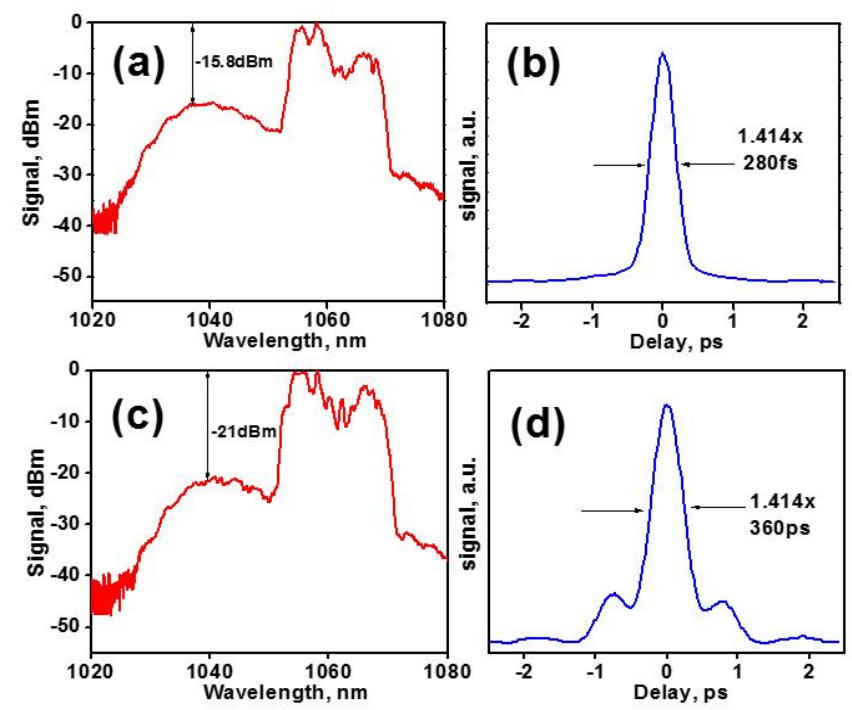

Figure 5: Optical spectrum and autocorrelation traces of power amplifier at (a) $7 \mathrm{~mW}$ and (b) $30 \mathrm{~mW}$ input signal strength.

Total $35 \mathrm{~W}$ of pump power is coupled to the inner clad of $\mathrm{Yb}$ DCF. The other end of Yb DCF was spliced with a high power fiber pigtailed collimator. The amplified signal was sent to the grating compressor. The compressor consists of a pair of gratings (groove density: 600 lines/mm) and a broad band retroreflector arranged in near littrow configuration. The overall compression efficiency of the grating pair was found to be $\sim 55 \%$. A part of the amplifier output was used to monitor the spectrum of the mode locked pulses.

In order to find the optimum signal strength, we first recorded the characteristics of the amplified pulses at a fixed pump power of $\sim 15 \mathrm{~W}$ with varying signal strength. It is observed that with increase in the input signal strength ASE contribution reduces whereas, in the time domain from the autocorrelation (AC) measurements it was found that the pulse gets distorted. Optical spectrum and AC traces of amplified 
pulses after the compression at two representative input signal strengths are shown in Fig 5. At low input signal strength $(\sim 7$ $\mathrm{mW})$, the ASE peak is $\sim 16 \mathrm{dBm}$ below the signal peak as shown in Fig. 5(a). In the linear scale ASE to signal peak is $\sim 3$ $\%$. The AC shows smooth and clean profile with FWHM 396 fs which corresponds to $\sim 280$ fs pulse duration assuming a Gaussian pulse profile as shown in Fig. 5 (b). With increase in the input signal strength to $\sim 30 \mathrm{~mW}$, the ASE peak is 21 $\mathrm{dBm}$ below the signal peak. In the linear scale ASE to signal peak is below $1 \%$. In the temporal domain, the pulse no longer remains clean and smooth as evident from the AC trace shown in Fig. 5(d) with an estimated pulse duration of 360fs.

Although, increasing the input signal strength to the power amplifier reduces the contribution from ASE, the signal enhancement in the pre-amplifier becomes practically disadvantageous due to strong SPM induced nonlinearity. Since grating pair compensates only the linear chirp, the residual nonlinear chirp distorts the pulse profile and results in enhanced pulse duration as well. Nonlinearity in the preamplifier is more significant as it consists of SMF (core diameter is small $\sim 6 \mu \mathrm{m}$ ). Thus, the signal strength in the preamplifier should be managed in such a way that it feeds the power amplifier sufficiently to keep ASE below 1\% of the amplified signal and simultaneously maintaining small SPM induced nonlinearity. Based on our measurement we found that an optimum input signal strength of $10 \mathrm{~mW}$ to get clean and smooth pulses from the power amplifier and at the same time keeping the ASE contribution low.
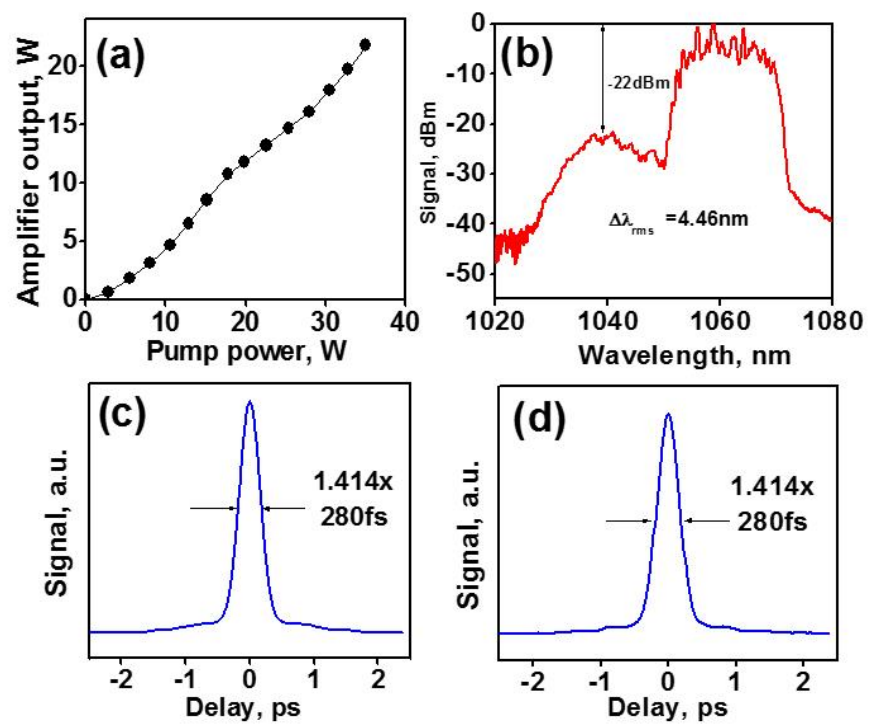

Figure 6: (a) Variation of amplifier output power with pump power coupled in Yb DCF. (b) Spectral profile of mode locked pulse after power amplification, (c) and (d) AC trace of the compressed amplified pulses at $1 \mathrm{~W}$ and $30 \mathrm{~W}$ pump power respectively.

Figure 6 shows the power amplification characteristics in Yb DCF amplifier with the optimum input signal strength. The amplified average power scales up almost linearly with the pump power. The variation of amplified power with the pump power is shown in Fig. 6(a). At the maximum coupled pump power of $35 \mathrm{~W}$, amplified signal power was measured to be $21.8 \mathrm{~W}$ corresponding to single pulse energy of $\sim 590 \mathrm{~nJ}$. The pump to signal power conversion efficiency was found to be $\sim 62 \%$. The recorded spectral profile of the mode locked pulses after power amplification is shown in Fig. 6(b). It can be seen that there is no significant contribution from amplified spontaneous emission and the rms spectral width was calculated to be 4.46 nm. Figure 6(c) and 6(d) show the AC traces of the amplified pulses after compression into grating pair at low and high pump powers. It can be seen that there is no appreciable change in the pulse shape and duration with increase in the pump power. The temporal profile is significantly smooth and free from any side lobes with a measured pulse duration of $280 \mathrm{fs}$ (assuming a Gaussian pulse shape). It is interesting to note that with optimum input signal strength the spectral and temporal profile of the pulses remain unaffected by the power amplification process within the operating range of the pump power.

\section{CONCLUSION}

In conclusion, we have studied the power amplification characteristics of all-fiber Yb-doped double clad fiber amplifier seeded by the pulses extracted after the NPR port of ANDi mode-locked Yb-doped fiber oscillator. 21.8 W of amplified average power at $37 \mathrm{MHz}$ repetition rate was obtained at pump power of $35 \mathrm{~W}$ corresponding to $\sim 62 \%$ pump to signal power conversion efficiency. The single pulse energy of the amplified mode locked pulses was $~ 590 \mathrm{~nJ}$. The effect of pre-amplifier signal strength on temporal and spectral profiles of amplified pulses were investigated experimentally. It was found that though stronger signal strength at the input of the power amplifier is useful to increase the threshold for onset of amplified spontaneous emission (ASE), however, it may lead to severe distortion in temporal profile after compression due to enhanced nonlinearity. We conclude that there is an optimum signal strength for generation of clean and smooth ultrashort pulses with low ASE contribution with in the operating range of the power amplifier. The compressed pulse duration of the amplified pulses was measured to be 280 fs.

\section{References}

[1]. A. Chong, J. Buckley, W. Renninger and F. Wise, “Allnormal-dispersion femtosecond fiber laser,” Opt. Exp., 14(21), 10095-10100, 2006.

[2]. A. Chong, W. Renninger and F. Wise, "All normal dispersion femtosecond fiber laser with pulse energy above 20nJ,” Opt. Lett. 32(16), 2408-10, 2007.

[3]. A. Chong, W. Renninger and F. Wise, J. Opt. Soc. Am. B. 25, 140 (2008).

[4]. K. Kiew, W. Renninger, A. Chong and F. Wise, "Sub

100fs pulses at Watt level powers from a dissipative soliton fiber laser,” Opt. Lett. 34(5), 593-95, 2009. 
[5]. P. K. Mukhopadhyay, K. Özgören, İ. L. Budunoğlu, F. Ö. İlday, "All-fiber low-noise high-power femtosecond Yb-fiber amplifier system seeded by an all-normal dispersion fiber oscillator,” IEEE J. Sel. Top. Quant. 15(1), 145-152, 2009. [6]. P. K. Mukhopadhyay, P. K. Gupta, K. S. Bindra, and S. M. Oak, "Note: Amplification characteristics of all-normal- dispersion mode-locked $\mathrm{Yb}$-doped fiber laser: Influence of input pulse shape,” Rev. Sci. Instrum. 84, 076107, 2013. [7]. H. CoIc, “Analytic modelling of high-gain ytterbiumdoped fibre amplifiers,” J. Opt.A 4(2), 120-129, 2002.

[8]. Trial version of the simulation software is available at www.liekki.com 